\section{Management of right main bronchial rupture with a double lumen endotracheal tube in a patient with blunt chest trauma}

\author{
Seung Hwan Seol', Woon Jeong Lee', Seon Hee Woo' ${ }^{1}$, Dae Hui Kim¹, \\ Jong Hui Suh ${ }^{2}$ \\ 'Department of Emergency Medicine, Incheon St. Mary's Hospital, The Catholic University of Korea College \\ of Medicine, Incheon, Korea \\ ${ }^{2}$ Department of Thoracic Surgery, Incheon St. Mary's Hospital, The Catholic University of Korea College of \\ Medicine, Incheon, Korea
}

Tracheobronchial disruption is one of the most severe injuries caused by blunt chest trauma. It may cause airway obstruction and resulting life-threatening respiratory deficiency. However, the clinical presentations are variable and frequently difficult to diagnose. We report a case of a previously healthy 16 -year-old man with complete right main bronchial transection sustained after a vehicular accident, who had progressive dyspnea, subcutaneous emphysema in the neck and anterior chest wall, and bilateral tension pneumothorax. Prompt chest tube drainage for suspected bilateral tension pneumothorax and a tracheal intubation were performed. Shortly after the positive pressure ventilation, severe subcutaneous emphysema developed and he was at risk for developing shock. Additional chest tubes were inserted. An emergency bronchoscopy showed rupture of the right main bronchus. After changing to a double lumen endotracheal tube, the patient's condition improved. A surgical closure was performed and postoperative bronchoscopy showed good repair. The patient was discharged without complications.

Keywords Thoracic injuries; Bronchial rupture; Double-lumen endotracheal tube

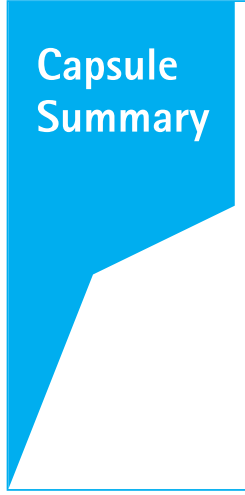

What is already known

Tracheobronchial rupture from blunt chest trauma is potentially lethal.

What is new in the current study

After intubation, we used portable bronchoscopy to identify a main bronchial rupture and changed the intubation tube to a double lumen intubation tube, which improved the patient's clinical condition.
eISSN: 2383-4625

Received: 9 September 2017

Revised: 15 October 2017

Accepted: 31 October 2017

Correspondence to: Woon Jeong Lee Department of Emergency Medicine, Incheon St. Mary's Hospital, The Catholic University of Korea College of Medicine, 56 Dongsu-ro, Bupyeong-gu, Incheon 21431, Korea

E-mail: Limleeem@catholic.ac.kr

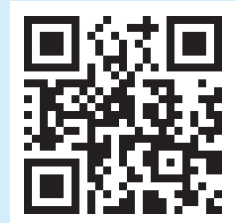

How to cite this article:

Seol SH, Lee WJ, Woo SH, Kim DH, Suh JH. Management of right main bronchial rupture with a double lumen endotracheal tube in a patient with blunt chest trauma. Clin Exp Emerg Med 2017;4(4):250-253.

This is an Open Access article distributed under the terms of the Creative Commons Attribution Non-Commercial License (http:// creativecommons.org/licenses/by-nc/4.0/). 


\section{INTRODUCTION}

Delayed diagnosis of tracheobronchial injury due to blunt chest trauma may occur because the condition is rare and difficult to diagnose. 'The time to diagnosis can directly affect patient survival; inaccurate diagnosis or inappropriate treatment may result in a life-threatening condition. ${ }^{1,2}$ We report a literature review and describe our experience with a patient with a right main bronchus rupture which was initially misdiagnosed as tension pneumothorax, despite the fact that chest computed tomography (CT) was performed. Afterward, an emergency bronchoscopy was performed and a double lumen endotracheal tube was used to rectify the incorrect treatment.

\section{CASE REPORT}

A 16-year-old man was struck by a car while riding a bicycle and was taken to the emergency room with chief complaints of chest pain and dyspnea. He was mentally alert and his vital signs included a blood pressure of $130 / 83 \mathrm{mmHg}$, pulse of 93 beats per minute, respiratory rate of 22 breaths per minute, body temperature of $36.6^{\circ} \mathrm{C}$, and $90 \%$ oxygen saturation. The patient presented with an ill appearance, decreased respiratory sounds in both lungs, and acute pain in his right chest. From the portable cervical lateral view, deep neck subcutaneous emphysema was observed in the soft tissues of the anterior cervical spine; chest images revealed subcutaneous emphysema in the right chest. Chest CT was performed immediately after confirming that the patient's paroxysmal events appeared to be stable. From the axial view, mediastinal emphysema was observed in the right lung contusion and main bronchus areas along with a small amount of bilateral pneumothorax in the apex of the lung (Fig. 1A). The main bronchial laceration was clearly seen from the coronal view, but there was a mistake of not checking the view (Fig. 1B). Despite the oxygen supply, the patient complained about worsening dyspnea and developed cyanosis, diaphoresis, and palpitations along with symptoms of anxiety. His oxygen saturation level decreased to $80 \%$. The hypophonesis of both lungs appeared to have decreased, suggesting the progression of tension pneumothorax. An 18-gauge needle was used to perform emergency needle aspiration between the second and third ribs on both thoraces and chest tubes were inserted on both sides of the chest. However, the oxygen saturation level still did not improve and the deep neck subcutaneous emphysema in the cervical spine and chest wall were aggravated; therefore, an endotracheal tube was inserted, after which gas analysis of the arterial blood revealed a $\mathrm{pH}$ of $7.29, \mathrm{pO}_{2}$ of $69 \mathrm{mmHg}$, $\mathrm{pCO}_{2}$ of $51 \mathrm{mmHg}$, and $91 \% \mathrm{O}_{2}$ saturation. Despite the endotracheal tube, the oxygen saturation did not improve and the subcutaneous emphysema progressed. Conscious sedation was performed and a ventilator was attached; however, evacuation of air through the chest tubes remained poor and the oxygen levels did not fully recover. Subsequently, a self-inflating bag was used to supply oxy-
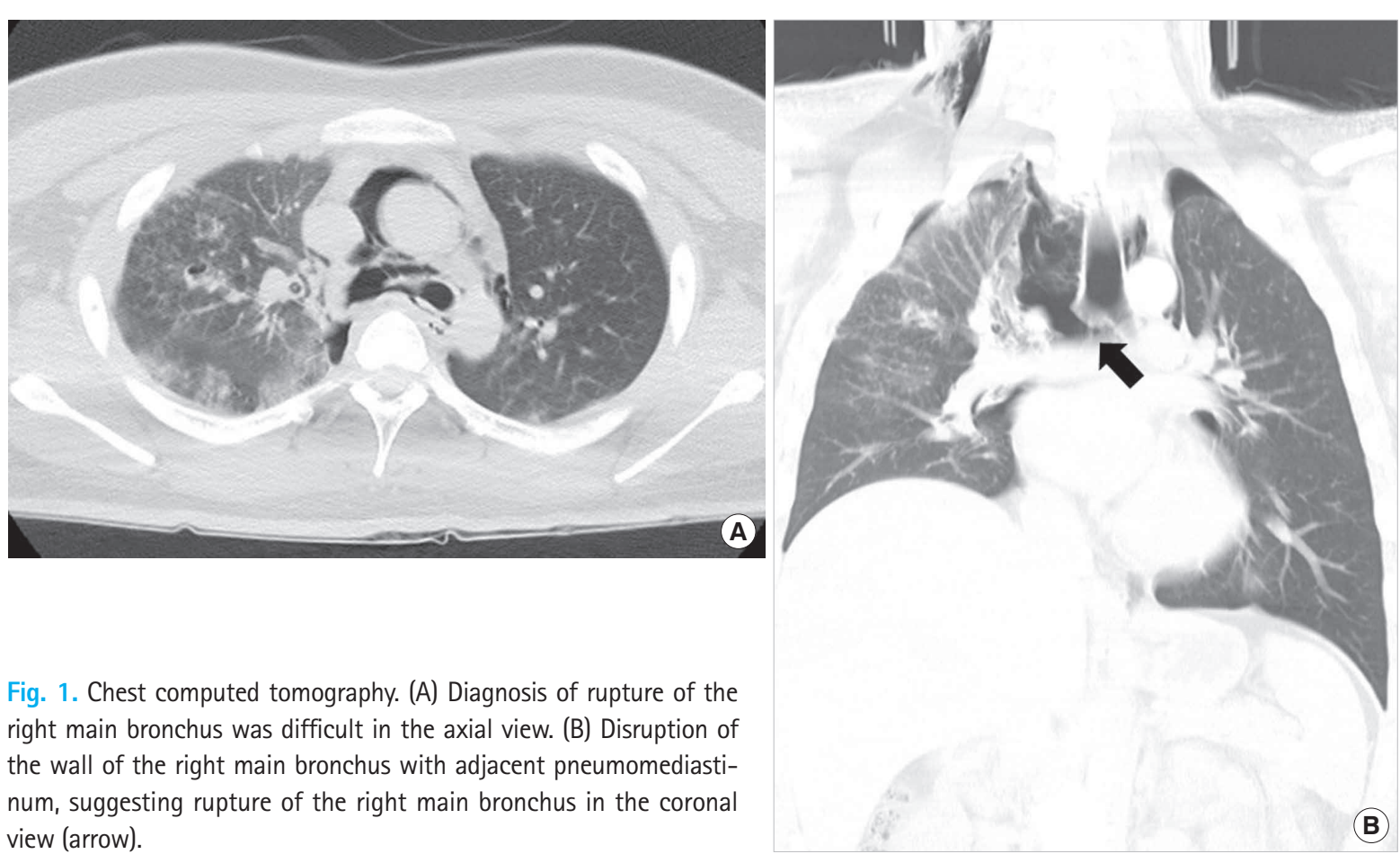

Fig. 1. Chest computed tomography. (A) Diagnosis of rupture of the right main bronchus was difficult in the axial view. (B) Disruption of the wall of the right main bronchus with adjacent pneumomediastinum, suggesting rupture of the right main bronchus in the coronal view (arrow). 


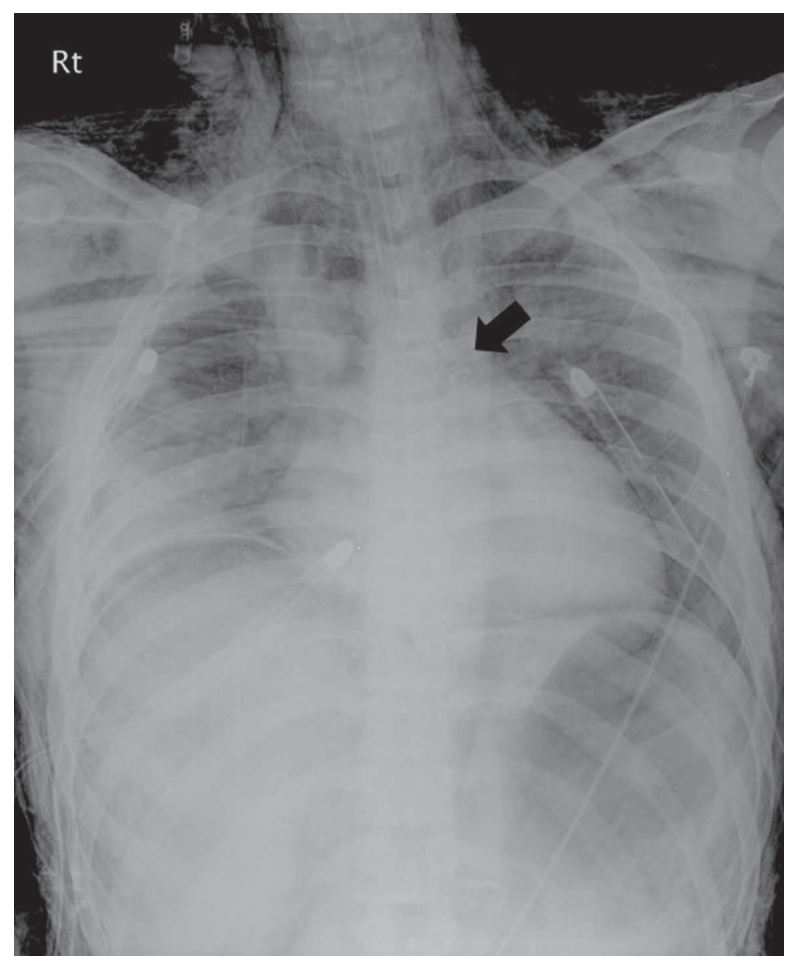

Fig. 2. Chest X-ray. The tip of the double lumen endotracheal tube is located in the left main bronchus (arrow). Three chest tubes were inserted on both sides of the chest cavity.

gen. Despite this, the air deflation was not smooth and there was large resistance to the ventilations. Suspecting an error in the placement and the function of chest tubes, a third tube was inserted in the intercostal space between the fifth and the sixth ribs on the right side. The patient's oxygen level briefly rose to $90 \%$, but the resistance during bagging persisted. A suction tube was inserted but did not go deeper than $25 \mathrm{~cm}$ from the incisor. In order to identify the bronchial injury, a portable bronchoscopy was performed. The endotracheal ends were almost blocked by blood clots. A guide wire was used to remove the blood clots and a right bronchus proximal rupture was confirmed. A double lumen intubation tube (35-Fr left Broncho-Cath, Mallinckrodt, St Louis, MO, USA) was then inserted to the left bronchus (Fig. 2). The patient's oxygen level increased to $95 \%$ and his other vital signs stabilized. The patient was referred to the thoracic surgery department. He was hospitalized in the intensive care unit and an emergency operation was performed. During the operation, a right bronchus rupture was discovered $2 \mathrm{~cm}$ directly under the carina. An end-toend anastomosis with interrupted suture was performed using 3.0 Vicryl. The patient recovered after the operation and was discharged from the hospital after 21 days without complications.

\section{DISCUSSION}

Tracheobronchial rupture from blunt chest trauma is a potentially lethal injury. ${ }^{1}$ Although its precise frequency is unknown, it is estimated to occur in $0.03 \%$ to $2.5 \%$ of cases, a rate less frequent than that of other thoracic damage..$^{1-3}$ The physiological cause of tracheobronchial rupture can be attributed to high-speed impact and sudden deceleration, which in turn decreases the diameter of the thoracic cavity and increases the internal bronchial pressure while the epiglottis is closed, which may rupture or tear the trachea. ${ }^{1,2}$ Car accidents are the most common cause of tracheobronchial ruptures in adults. ${ }^{2}$ For children between 5 and 6 years of age, car accidents and falls are the main causes, while bicycle accidents are the main cause in children 12 to 14 years of age. ${ }^{4}$ Injuries within $2 \mathrm{~cm}$ of the carina and of the right main bronchus occur in $76 \%$ and $43 \%$ of patients, respectively. ${ }^{2,3}$ While rupture of the right main bronchus can be diagnosed earlier than a leftsided rupture, it has a higher mortality rate. ${ }^{2}$ The clinical features of tracheobronchial injury include air leakage, mediastinal emphysema, deep neck subcutaneous emphysema, hemoptysis, pneumothorax, serious atelectasis, and failure of lung re-expansion after chest tube insertion. ${ }^{4}$ Other physical, imaging-related findings include shock, cyanosis, rib fracture, pulmonary contusion, etc. ${ }^{5,6}$

A strong suspicion based on clinical symptoms and medical imaging findings is important for diagnosis. Simple chest radiographs are not sufficient to identify tracheobroncheal rupture but are useful to confirm mediastinal emphysema and pneumothorax. ${ }^{5,6} \mathrm{~A}$ radiographic air gradient observed in the cervical lateral view is a reason to suspect air leak from under the deep cervical fasciae. Bilateral pneumothoraces suggests the possibility of tracheal laceration. The upper boundary of collapsed lung falls below the height of the ruptured bronchus; the so called "fallen lung sign" is characteristic of a complete rupture of the bronchus. However, absence of these signs does not mean that the disease is not present..$^{6-8}$ Continuous air leak from bronchial injury should be suspected if there is difficulty evacuating air after chest tube insertion and the lung fails to re-inflate. ${ }^{8}$ Similarly, in our case, the patient had difficulty evacuating air after chest tube insertion and the lung did not re-inflate. These signs were initially considered to indicate a failed chest tube insertion and an additional chest tube was inserted, which exacerbated the dyspnea and deep neck subcutaneous emphysema. Moreover, large resistance to the self-inflating bag resulted in an insufficient supply of oxygen. The emergency bronchial endoscope revealed respiratory obstruction due to blood clotting in the trachea and bronchus. Be- 
cause blood clots from the main bronchus rupture obstructed the trachea, not enough oxygen was supplied to the lungs and it was unclear how much air was leaking from the chest tube. Consequently, the main bronchus rupture was not suspected early on, and treatment was delayed due to several attempts to insert chest tubes. A prompt decision to replace the endotracheal tube could not be made due to the serious progression of the deep neck subcutaneous emphysema in the face and cervical spine. After confirming the presence of atresia of the trachea with flexible bronchoscopy, a double lumen endotracheal tube was inserted as a replacement and lung ventilation was performed in the left lung. Bronchoscopy is the most accurate tool for definite diagnosis of trachea-bronchial rupture. ${ }^{8,9}$ All patients with blunt chest trauma and those with consistent air leaks should receive immediate bronchoscopy. ${ }^{9-11}$

In some cases, patients are not in a condition to safely perform bronchoscopy and blood and mucus may accumulate in the damaged bronchus, which hinders accurate diagnosis. ${ }^{9}$ Additionally, bronchoscopy is not always available in all emergency rooms. CT is a noninvasive test that can provide useful information to understand the cause of chest trauma without definite diagnosis through bronchoscopy. ${ }^{7.8}$ However, the presence of surrounding air in the trachea and bronchus from deep neck subcutaneous emphysema often blocks the view of the bronchus wall structure and contours. ${ }^{9}$ Both sagittal views and coronal reconstructions should be checked in order to confirm tracheobronchial injury. ${ }^{8}$ In our case, chest CT was performed early, but there was difficulty in observing the bronchus rupture in the axial view; the bronchus rupture was later confirmed from the coronal view (Fig. 1B).

Selective treatment of tracheobronchial rupture includes anastomosis through primary closure. ${ }^{10,11}$ This primary closure helps maintain lung and respiratory functions in 90\% of cases. Premature closure is the best way to prevent infection, scarring, and stricture complications..$^{10,11}$ Unrecognized rupture in the main bronchus may cause serious complications and ultimately lead to death. ${ }^{11}$ Conservative treatment may be considered for patients with damage that affects less than one-third of the tracheobronchial diameter, those with lungs that have been re-inflated properly after chest tube insertion, and those with limited air leakage through the tube."

Emergency physicians should be aware of the potential for main bronchus injury when there is prolonged dyspnea after chest tube insertion or continued air leak in blunt chest trauma patients with pneumothorax and mediastinal emphysema. Immediate action should be taken by using a double lumen endotracheal tube to perform selective one-lung ventilation.

\section{CONFLICT OF INTEREST}

No potential conflict of interest relevant to this article was reported.

\section{REFERENCES}

1. Cassada DC, Munyikwa MP, Moniz MP, Dieter RA Jr, Schuchmann GF, Enderson BL. Acute injuries of the trachea and major bronchi: importance of early diagnosis. Ann Thorac Surg 2000;69:1563-7.

2. Mussi $A$, Ambrogi MC, Ribechini A, Lucchi M, Menoni $F, A n-$ geletti CA. Acute major airway injuries: clinical features and management. Eur J Cardiothorac Surg 2001;20:46-51.

3. Bingol-Kologlu $M$, Fedakar $M$, Yagmurlu $A$, Fitoz $S$, Dindar $H_{\text {, }}$ Gokcora IH. Tracheobronchial rupture due to blunt chest trauma: report of a case. Surg Today 2006;36:823-26.

4. Kaptanoglu M, Dogan K, Nadir A, et al. Tracheobronchial rupture: a considerable risk for young teenagers. Int J Pediatr Otorhinolaryngol 2002;62:123-8.

5. Moore MA, Wallace EC, Westra SJ. Chest trauma in children: current imaging guidelines and techniques. Radiol Clin North Am 2011;49:949-68.

6. Nishiumi N, Maitani F, Yamada S, et al. Chest radiography assessment of tracheobronchial disruption associated with blunt chest trauma. J Trauma 2002;53:372-7.

7. Van Hise ML, Primack SL, Israel RS, Muller NL. CT in blunt chest trauma: indications and limitations. Radiographics 1998;18: 1071-84.

8. Wintermark M, Schnyder P, Wicky S. Blunt traumatic rupture of a mainstem bronchus: spiral CT demonstration of the "fallen lung" sign. Eur Radiol 2001;11:409-11.

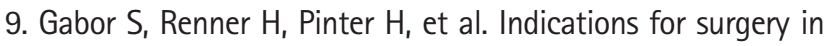
tracheobronchial ruptures. Eur J Cardiothorac Surg 2001;20: 399-404.

10. Juvekar NM, Deshpande SS, Nadkarni A, Kanitkar S. Perioperative management of tracheobronchial injury following blunt trauma. Ann Card Anaesth 2013;16:140-3.

11. Kiser AC, O'Brien SM, Detterbeck FC. Blunt tracheobronchial injuries: treatment and outcomes. Ann Thorac Surg 2001;71: 2059-65. 